\title{
Integrated Electronic Discharge Summaries- Experience of a Tertiary Pediatric Institution
}

\author{
Daryl R. Cheng ${ }^{1,2}$ Merav L. Katz ${ }^{1}$ Mike South ${ }^{1,2}$ \\ ${ }^{1}$ EMR Project Team, The Royal Children's Hospital Melbourne, \\ Parkville, Australia \\ 2 Department of General Medicine, The Royal Children's Hospital \\ Melbourne, Parkville, Australia
}

Address for correspondence Daryl R. Cheng, Department of General Medicine, The Royal Children's Hospital Melbourne, 50 Flemington Road, Parkville, VIC 3052, Australia (e-mail: daryl.cheng@rch.org.au).

Appl Clin Inform 2018;9:734-742.

\section{Abstract \\ Keywords \\ - best practice \\ - discharge summaries \\ - electronic medical records \\ - template}

Objective Succinct and timely discharge summaries (DSs) facilitate ongoing care for patients discharged from acute care settings. Many institutions have introduced electronic DS (eDS) templates to improve quality and timeliness of clinical correspondence. However, significant intrahospital and intraunit variability and application exists. A review of the literature and guidelines revealed 13 key elements that should be included in a best practice DS. This was compared against our pediatric institution's eDS template-housed within an integrated electronic medical record (EMR) and used across most inpatient hospital units.

Methods Uptake and adherence to the suggested key elements was measured by comparing all DSs for long stay inpatients (> 21-day admission) during the first year of the EMR eDS template's usage (May 2016-April 2017).

Results A total of 472 DSs were evaluated. Six of 13 key elements were completed in $>98.0 \%$ of DSs. Conversely, only $<5.0 \%$ included allergies or adverse reaction data, and $<11.0 \%$ included ceased medications or pending laboratory results. Inclusion of procedure information and pending laboratory results significantly improved with time ( $p=0.05$ and $p<0.04$, respectively), likely as doctors became more familiar with EMR and autopopulation functions. Inclusion of "discharge diagnosis" differed significantly between medical ( $n=406 / 472 ; 99.0 \%$ ) and surgical ( $n=32 / 472 ; 51.6 \%$ ) DSs.

Conclusion Uptake and adherence to an EMR eDS template designed to meet best practice guidelines in a pediatric institution was strong, although significant improvements in specific data elements are needed. Strategies can include a modification of existing eDS templates and junior medical staff education around best practice.

\section{Background and Significance}

Effective ongoing management of patients discharged from acute care settings is facilitated via succinct and timely discharge summaries (DSs). ${ }^{1-3}$ Transitions of care at points such as these have been identified as a time of increased risk for patient adverse events. ${ }^{4}$ Thus, many health care institutions have introduced standardized electronic DS (eDS) not only to improve the quality and timeliness of clinical corre- spondence, but also to prevent breakdown of communication between acute and primary care providers. ${ }^{5-7}$

The integration of technology with DS has been driven by clear enhancements in the quality and transmission of DS. These include improved legibility, consistency, accessibility, security, accuracy, and inclusion of key information and timeliness. $^{2,8-13}$ eDS are also more likely to include discussion of follow-up issues and pending test results compared with written DS. ${ }^{12}$ In addition to the potential safety and quality received

April 18, 2018

accepted after revision

July 29, 2018 @ 2018 Georg Thieme Verlag KG
Stuttgart · New York
DOI https://doi.org/

10.1055/s-0038-1669461. ISSN 1869-0327. 
benefits, there is evidence to suggest that eDS results in higher primary care clinician and patient satisfaction-not least because structured eDS organized by subheadings result in better readability compared with a lengthy narrative.,14-19

eDSs are currently generated using a variety of technology options ranging from standalone rich text format solutions to modules within integrated electronic medical records (EMRs). ${ }^{20}$ eDSs are then either physically or electronically dispatched to key stakeholders based on end user preference. However, significant intrahospital and intraunit variability in DS content still exists. To address this, some countries have released recommendations regarding health information transmission requirements, although these do not contain significant detail. ${ }^{21}$ Others have gone one step further and created national guidelines for DS. ${ }^{22,23}$

Within the Australian context, there have been numerous strategies to address this at all levels-including national guidelines developed by the Australian Commission on Safety and Quality in Health Care (ACSQHC). ${ }^{7}$ These aim to standardize content and structure of eDS across a variety of clinical settings, in anticipation of the expected increased availability of eDS within the national personalized medical record system (My Health Record) in the near future. ${ }^{24}$

The Royal Children's Hospital Melbourne (RCH), is an example of a local application of eDS templates as part of an integrated comprehensive EMR system (EpicCare, Madison, Wisconsin, United States)-which was launched in April 2016. Since the EMR went live, there has been a standardized template for eDS across most units and areas of the hospital. The template includes autopopulated patient demographic and care information, including diagnoses and procedures. Furthermore, it allows for the inclusion of automatic refreshing links, which pull key data elements from various sections of the patient's record (e.g., discharge medications and appointments), as well as free text ( - Fig. 1 ). Although users can delete certain elements, sections, or even bypass the template and write free text only, this is strongly discouraged to encourage consistency of practice.

Prior to the commencement of the RCH EMR, medical staff were given standardized EMR training by two trainers, including orientation to the DS template. To improve usage and content reliability, two additional refresher sessions were offered to all trainees at 3 and 6 months posttemplate implementation. This was coupled with quick reference printed and electronic resource guides embedded within the EMR available at user discretion.

\section{Objective}

Given many tertiary institutions are transitioning toward EMR, it is imperative that features within this process are revised, audited, and optimized. Thus, this study aims to determine best practice pediatric DS guidelines via a literature review and to then evaluate the existing RCH eDS template against these guidelines. The secondary aim is to assess the real-time uptake and use of DS templates by doctors within an Australian tertiary academic pediatric institution. By identifying potential barriers to both the development and adherence to best practice templates, future areas for improvement and research could be identified for local, national, and international contexts.

\section{Methods}

\section{Literature Review and Analysis}

Suggested DS key elements from ACSQHC national guidelines are predominantly based on research and consultation within the adult population. ${ }^{7}$ Thus, an additional literature review was performed to ensure that pertinent criteria relevant specific to the pediatric population were not excluded (e.g., immunizations given during hospitalization). ${ }^{18}$

This was conducted via a comprehensive search of Medline Ovid, EMBASE, and the Cochrane Library in April 2017 for articles relating to DS, with the search criteria limited to English language and human subjects (-Appendix A). The initial search identified 291 articles. Following removal of duplicates, screening of titles and abstracts, and review of full-length articles, 35 articles were analyzed by two authors (M.K. and D.C.) and included in our study (- Fig. 2).

Key elements across the 35 articles were compared and discussed with an institutional expert consultative committee. Their task was to use existing evidence to create best practice guidelines with particular focus on developing key inclusion elements. A list of 13 elements constituting a "bestpractice" DS was developed, including: (1) patient details, (2) admission diagnosis, (3) discharge diagnosis, (4) investigations (e.g., laboratory tests, imaging tests), (5) procedures (surgical and other interventional procedures), (6) hospital course, (7) allergies, (8) adverse reactions, (9) ceased medications, (10) discharge medications, (11) pending laboratory results, (12) medical problems at discharge, and (13) followup details.

This was followed by a comparison against our institution's eDS practice, in the form of a retrospective cohort analysis assessing the DS of long stay patients (> 21-day inpatient stay) at RCH during the first year of its integrated EMR and eDS template (May 2016-April 2017) (-Fig. 3). Each eDS was analyzed and a comparison made between user-included and best-practice DS elements. The primary outcome measure was data available for each key criteria, whether auto- or manually populated.

Ethics approval was obtained from the RCH Human Research Ethics Committee (HREC \#DA001-2017-102).

\section{Cohort Selection}

Long stay inpatients were the target population selected for analysis. In pediatric settings, the bulk of inpatient encounters occur in otherwise well children with minimal comorbidities and only short admission periods. Thus, long stay patients are more likely to have multiple and complex comorbidities, medications, treatments, or follow-upresulting in the need for a more detailed and robust DS which would include DS key elements. DS from units that do not use the standardized discharged-from-hospital eDS template were excluded (mental health unit). Furthermore, DS relating to day-stay patients were not generated. eDSs were 


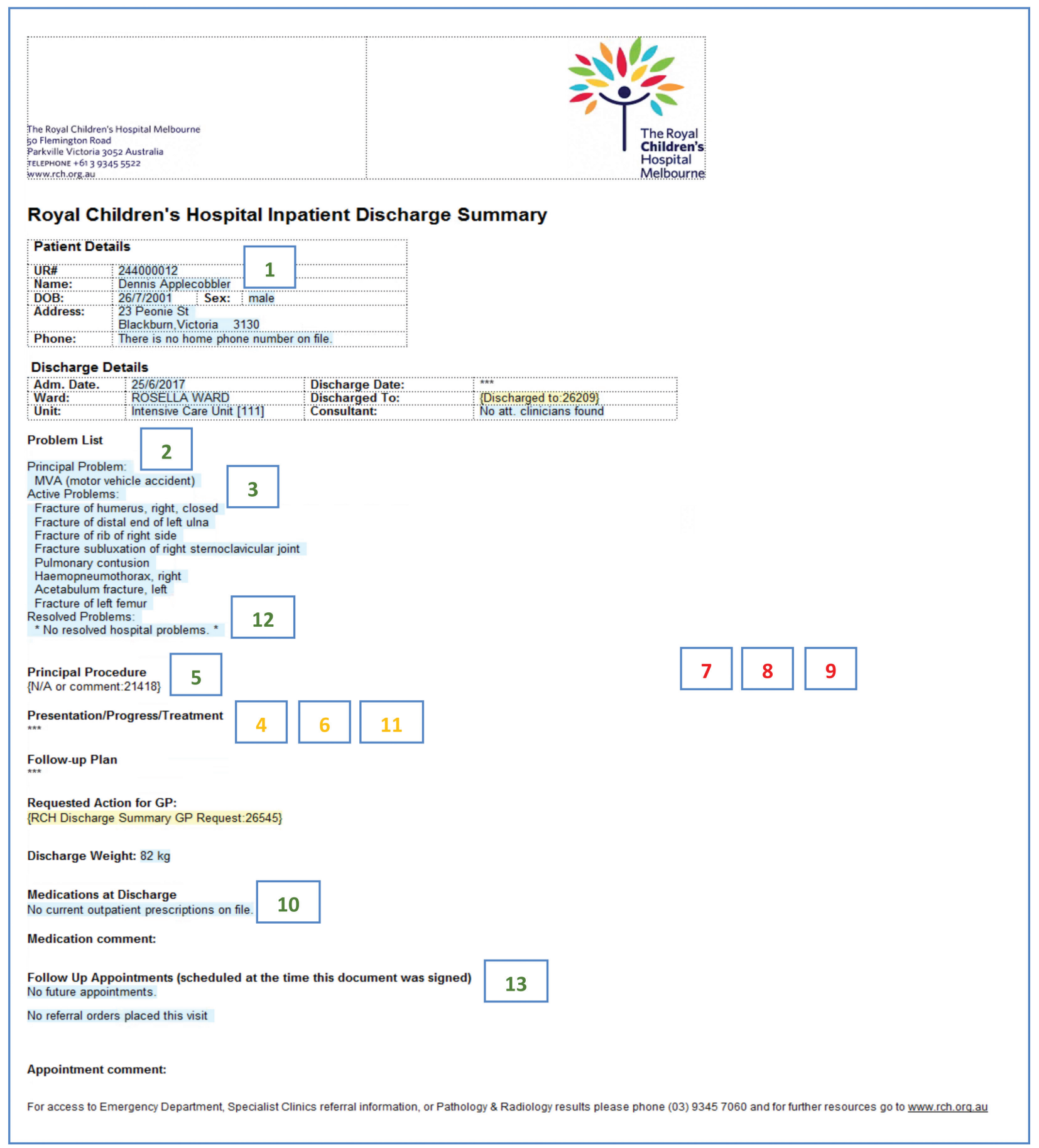

Fig. 1 Sample Royal Children's Hospital Melbourne electronic discharge summary template. Green numbers = autopopulated. Red numbers = no defined headings. Orange numbers = users were prompted to enter information. Users could enter free text information at their discretion.

reviewed and patient demographic data including age, sex, and inpatient unit was collected for each patient.

\section{Statistical Analysis}

Descriptive statistics were used to summarize the inclusion of best-practice DS elements; this was recorded by frequency and percentage. $t$-Test and chi-squared tests were used to compare inclusion of best-practice DS elements over time, and between medical and surgical subgroups. A $p$-value of $<0.05$ was considered significant.

\section{Results}

\section{RCH eDS Template}

Of the 13 best practice elements, 7 were autopopulated within the RCH DS template (-Fig. 1, numbered in green). Four other elements had the potential to be autopopulated, but were not customized nor included in the template at the time of the study. There were no defined headings for 3 of the 13 elements ( - Fig. 1, numbered in red). Users were prompted to enter patient-specific information for a further 


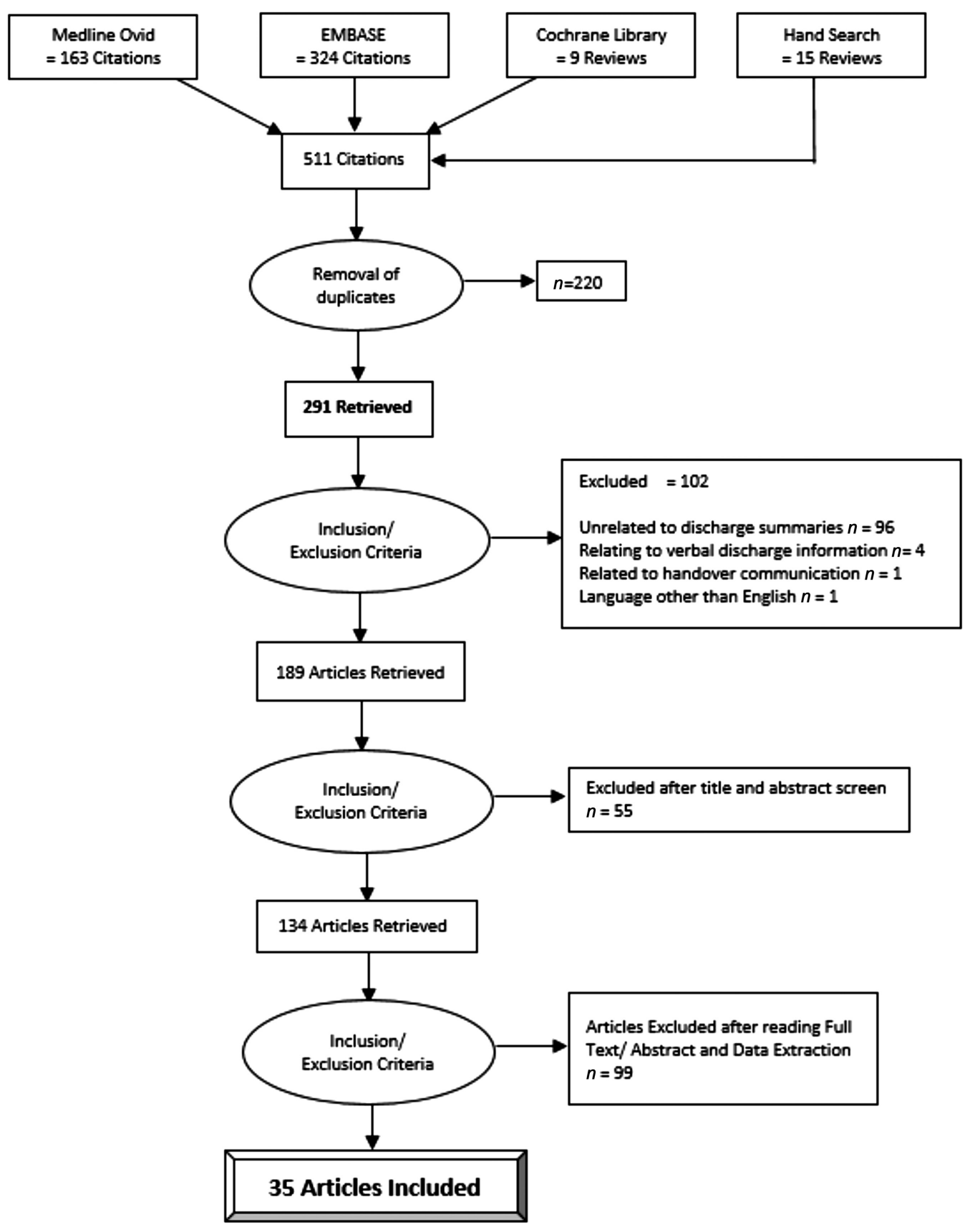

Fig. 2 Literature review algorithm.

3 elements (-Fig. 1, numbered in yellow) and they could enter free-text information for any other key elements at their discretion.

\section{User Uptake and Usage}

Following review of EMR for long stay patients, 564 patient episodes were identified. Note that 472 had DS available for evaluation. Of the DS available, $86.86 \%$ ( $n=410 / 472)$ were medical patients. Ninety-two DS were not generated as these related to administrative long stay patients and were appropriately excluded-continuous multi-day visit patients who were receiving outpatient-based treatments such as chemotherapy or rehabilitation.
Six of 13 best practice elements were completed in $>98.0 \%$ of DS: patient details $(N=472 / 472,100 \%)$, admission diagnosis $(N=472 / 472,100 \%)$, hospital course $(N=472 / 472,100 \%)$, discharge medications $(N=463 / 472$, $98.09 \%)$, medical problems at discharge $(N=468 / 472$, $99.15 \%$ ), and follow-up details ( $N=472 / 472,100 \%)$. The 7th autopopulated element-principal procedure-required users to enter N/A or comment on the procedure and was included in $92.37 \%$ of DS $(N=436 / 472)$. DS infrequently included allergies, adverse reaction data, ceased medications, and pending laboratory results (-Table 1 ).

Inclusion of procedure information and pending laboratory results improved with time, that is, from the first 

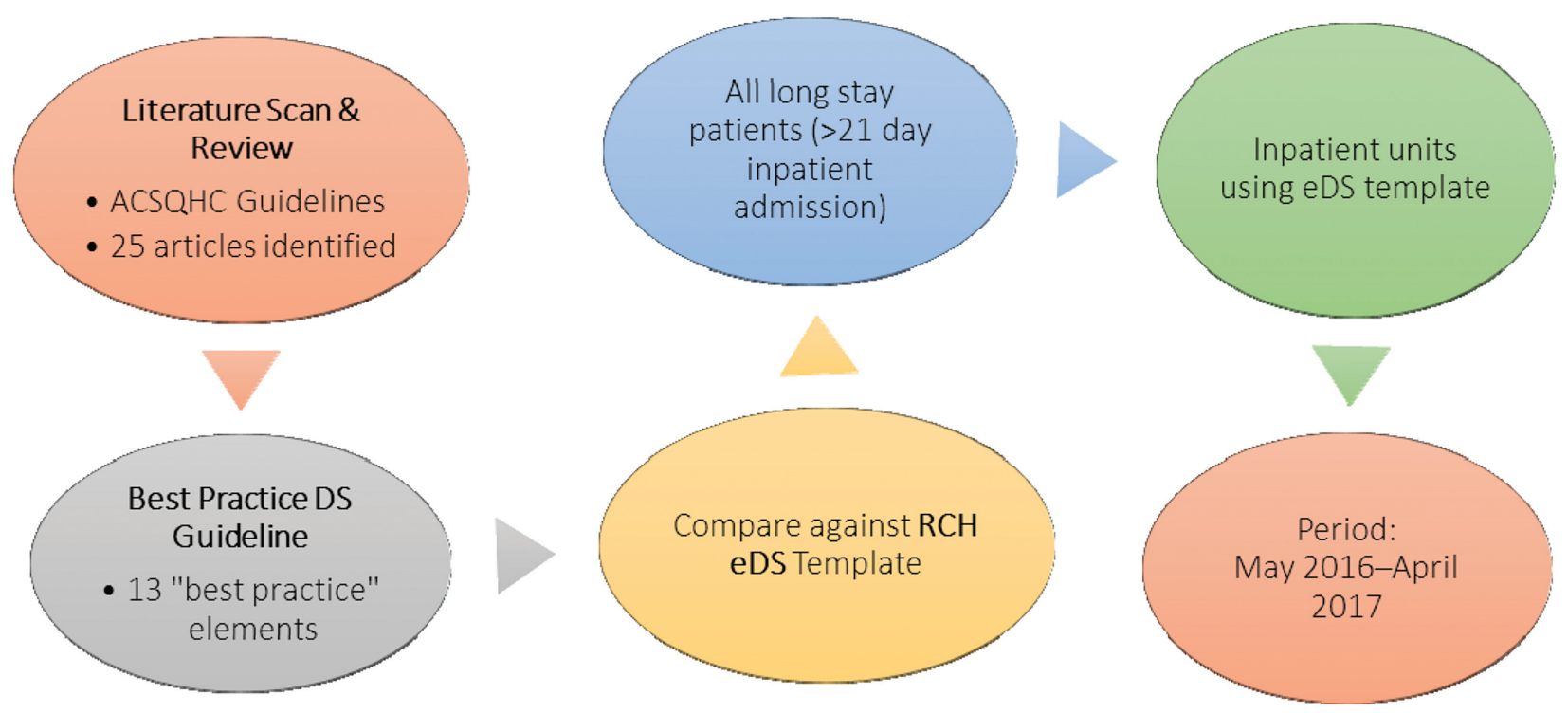

Fig. 3 Study methods algorithm. ACSQHC: Australian Commission on Safety and Quality in Health Care.

3-month period, May to July 2016, to the final 3-month period, February to April 2017 ( $n=77 / 86,89.53 \%$ to $n=142 / 148,95.95 \%, p=0.05$ and $n=4 / 86,4.65 \%$ to $n=24 / 148,16.22 \%, p=0.04$, respectively) (-Fig. 4). Though not statistically significant, there was an overall numerical improvement in inclusion of the pending investigations over time. Information around immunizations administered during a hospital stay was not included in DS unless specifically entered by end users.

Medical DS were significantly more likely to include a discharge diagnosis compared with surgical DS ( $n=406$ / $410,99.02 \%$ vs. $n=32 / 62,51.61 \%$, respectively). Furthermore, the discharge weight, an autopopulated feature spe- cific to the RCH DS, was included in $n=403 / 410,98.29 \%$, of medical DS and $n=62 / 62,100 \%$, of surgical DS.

\section{Discussion}

This study looked at best practice guidelines for DS and its implementation and uptake in a single tertiary pediatric institution. To the authors' knowledge, this study is the first of its kind within an Australasian context.

There is unequivocal existing recognition that variability and inconsistencies among DS content may be a barrier to efficient patient care. ${ }^{19}$ Even with existing standardized guidelines, it is important to consider pediatric nuances

Table 1 Best practice DS elements and inclusions

\begin{tabular}{|c|c|c|}
\hline Best practice DS elements & $\begin{array}{l}\text { RCH DSs with included } \\
\text { best practice elements } \\
N(\%)\end{array}$ & Autopopulated \\
\hline \multicolumn{3}{|l|}{ Total, $n=472$} \\
\hline [1] Patient details & $472(100)$ & Yes \\
\hline [2] Admission diagnosis & $472(100)$ & Yes \\
\hline [3] Discharge diagnosis & $438(92.80)$ & Yes \\
\hline [4] Investigations & $439(93.01)$ & Yes \\
\hline [5] Procedures & $436(92.37)$ & Yes \\
\hline [6] Hospital course & $472(100)$ & No \\
\hline [7] Allergies & $11(2.33)$ & Potentially Yes - but not currently included in template \\
\hline [8] Adverse reactions & $19(4.04)$ & Potentially Yes - but not currently included in template \\
\hline [9] Ceased medications & $19(4.04)$ & Potentially Yes - but not currently included in template \\
\hline [10] Discharge medications & $463(98.09)$ & Yes \\
\hline [11] Pending laboratory results & $51(10.81)$ & Potentially Yes - but not currently included in template \\
\hline [12] Medical problems at discharge & $468(99.15)$ & Yes \\
\hline [13] Follow-up details & $472(100)$ & No \\
\hline
\end{tabular}

Abbreviations: DS, discharge summary; RCH, Royal Children's Hospital Melbourne. 


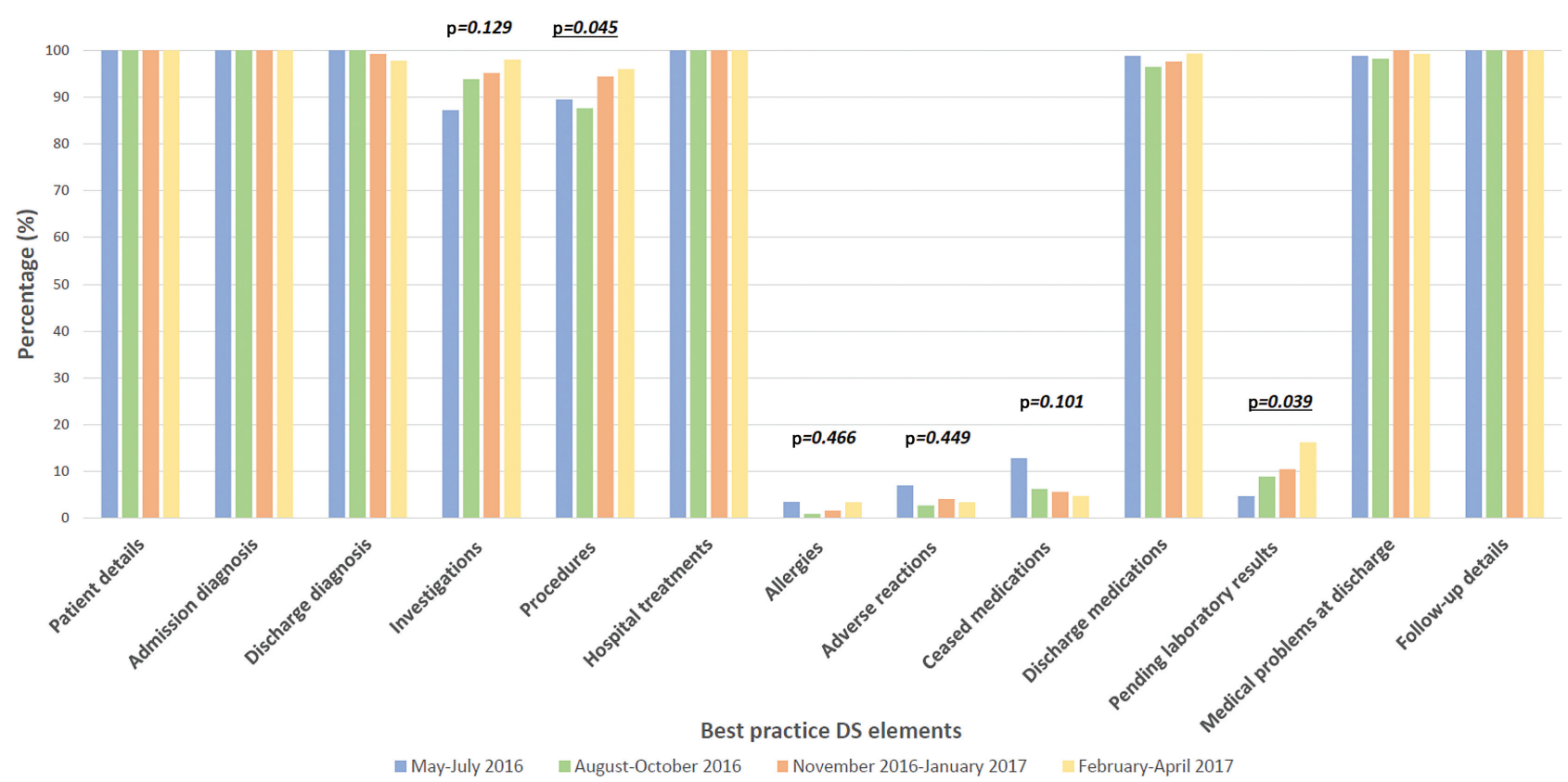

Fig. 4 Best practice elements included in Royal Children's Hospital Melbourne discharge summary (DS) (stratified by quarter).

including preventative measures and pediatric-specific information such as developmental history or social or schooling factors that may contribute to creating effective eDS and assist patient care for this cohort.

Examples of key elements relevant to both adult and pediatric population groups include patient demographics, admission and discharge diagnoses, hospital management, and treatment. Utilization of the autopopulation function in the design of our eDS templates was shown to significantly increase the likelihood of inclusion of these elements within each DS; consistent with previous studies. ${ }^{25-27}$ This is further evidenced by the poor uptake of best practice elements which were not autopopulated but only available for inclusion at the author's discretion-for example, allergies or adverse reaction data, ceased medications, or pending laboratory results.

Similar trends have been seen previously, with multiple studies finding diagnostic test results (33-63\%), hospital course (7-22\%), discharge medications (2-40\%), pending test results (65\%), patient or family counseling (90-92\%), and follow-up plans (2-43\%) are variable and often omitted elements in adult DS. ${ }^{9}$ This suggests that similar deficiencies in meeting best practice DS guidelines are likely present among other institutions, both adult and pediatric, within Australia.

Effectively developing or redesigning an eDS template could contribute to a significant improvement in the documentation of key information; including patient allergies, changes to medications, and specific requests of primary care providers or general practitioners on discharge. One way this can be achieved is by allowing for autopopulation or customization of suggested phrases and links. ${ }^{25,28}$ This reduces the need for double documentation and may have the flow on effect of increasing efficiency and timely delivery of DS to primary care providers. ${ }^{28-30}$ Ultimately, this may contribute to both referring provider satisfaction and improved patient outcomes as pertinent information is disseminated.
Movement into or out of hospital is a particularly vulnerable period for medication safety and potential prescribing errors. ${ }^{4,31}$ These are especially important in pediatrics where dose calculations are complicated and weight-based, and small errors can cause significant harm. ${ }^{32}$ Medicines administered during an inpatient stay including immunizations, adverse reactions, and ceased medications, either by autopopulation or manual user-entry, were sparsely included in DS that were evaluated . Besides a discharge medication list, selective autoinclusion of inpatient medications in eDS which are relevant to ongoing patient care could be beneficial for patient primary care providers. More importantly, ensuring these data are synchronized with other repositories or centralized databases (e.g., Australian Immunisation Register for immunization) will ensure seamless transition and recording of key patient information beyond the hospital stay.

Importantly, however, although these strategies may all lead to improvement in the inclusion of information within an eDS, the accuracy and validity of the information remains an arguably greater consideration and may have potential impact on patient care. Gattari et al have demonstrated that the introduction of EMRs along with eDS may unwittingly result in multiple sources of the same information within a patient's record-which in turn creates discrepancies between medication lists housed within an EMR and a similar list autopopulated to a DS. ${ }^{33}$ Others have also shown that $36.4 \%$ of DS in their health service contained some information errors. ${ }^{10}$ Errors in patients' DS have been correlated with increased rates of readmission to hospital and adverse patient outcomes.9,34-37

Among the features that EMR bring, comes the "copy and paste" or copy forward functionality. While this feature may support efficiency during clinical documentation, there is also the potential to promote inaccurate documentation that poses risks to patient safety. Tsou et al found that 66 to $90 \%$ of clinicians routinely use the copy and paste function. ${ }^{37}$ In 
cases where erroneous discharge information does not immediately impact patients, it may subsequently be detrimental to a patient at a later date-as incomplete and inaccurate information about a patient's hospitalization remains in their medical record and is rarely questioned once documented. ${ }^{38}$ To improve documentation reliability within EMRs, it is essential that documentation practices are monitored and routinely evaluated and assessed. ${ }^{39}$

Content reliability is also challenging because of interuser variability; even within our pediatric tertiary center where the implementation of eDS templates is almost universal. In most cases, it is the responsibility of junior medical staff to complete DS. There remained significant variability between various medical and surgical teams and departments. For example, surgical DSs would often use autopopulated procedure or operation names as a surrogate for discharge diagnoses, which was not always accurate. Inclusion of certain elements improved over time as clinicians presumably became more familiar with the integrated eDS/EMR system and also likely due to effects of education and familiarization sessions. Other contributing factors toward variability could include completion of DS by a clinical team rather than an individual clinician, time pressures associated with DS generation, and lack of clinical experience by junior doctors completing the DS. ${ }^{5}$

\section{Future Direction}

We propose several strategies to improve the quality of DS in the future. Our study targeted DS for long stay patients only, and within this cohort, deficiencies in meeting best practice standards were identified. Future research should include all inpatient units and patients (short or long stay) at the RCH to ensure best practice across all areas, departments, and patients is evaluated. More importantly, comprehensive future studies evaluating the accuracy of included eDS content are currently under way.

Work to optimize eDS templates has already begun, which involves modification of existing eDS templates to autopopulate best practice features, particularly around current deficit areas. Suggestions and prompts to enhance and simplify eDS completion are also being considered based on our findings. Guides around completing narrative portions of the DS are also being developed. Other principles useful in the implementation of an eDS are being considered, including the juxtaposition of allowing flexibility while ensuring key criteria are included. ${ }^{40}$ If found to improve the quality of eDS, these enhancements could be implemented within eDS across EMRs within Australia.

Training and education is a key plank in optimizing enduser usage of eDS technology and also best practice in DS completion. ${ }^{23}$ This helps to ensure that accurate and succinct patient data are first entered into the medical record, which is then autopopulated in eDS. Thus, when pertinent information is included, it is able to be utilized effectively by all clinicians involved in the patient's care.

Finally, strategies to develop eDS into a dynamic patient care tool should also be explored. Work in enabling two-way synchronization of key discharge information with national health records, health databases, disease registries, or other repositories will increase currency and accuracy of data across multiple information sources.

\section{Limitations}

This study examines inclusion of key data within eDS; however, it does not evaluate the accuracy of the information included as part of the autopopulation feature. Assessment of this is both user- and unit-dependent and is beyond the scope of this study. Nonetheless, it is a crucial piece for future research. Furthermore, our sample included long stay patients only and thus it is uncertain whether the deficits identified within DS are generalizable to short stay DS too.

\section{Conclusion}

DSs serve an important purpose of being the primary mode of communication between the inpatient and outpatient setting. Review of the literature revealed that variability and inconsistency exist within DS, and furthermore, that incomplete and erroneous completion can compromise patient care, safety, and follow-up. There is room for improvement in the completion of DSs across all specialties.

\section{Clinical Relevance Statement}

Discharge summaries remain the primary mode of communication between the inpatient and outpatient setting. Variability and inconsistency within discharge summaries, along with omitted key details and erroneous completion, can compromise patient care, safety, and follow-up. Regular optimization of electronic templates along with user training and education as well as synchronization with other dynamic databases can enhance effectiveness and reliability.

\section{Multiple Choice Questions}

1. Which key criteria is often omitted from pediatric discharge summaries?
a. Admission diagnosis.
b. Discharge medications.
c. Pending laboratory results.
d. Follow-up results.

Correct Answer: The correct answer is option c. Pending laboratory results and details around altered medications were included in $<10 \%$ of all discharge summaries. This creates a potential medication safety risk and/or duplication of therapy. By including them in discharge summaries, prevention of potential errors is maximized.

2. Which of these has been identified as a key issue with electronic discharge summary content?
a. Autopopulation of data.
b. Accuracy of content. 
c. Not including family details.

d. Inclusion of doctors' signature.

Correct Answer: The correct answer is option b. Accuracy of content has been identified as a major challenge in electronic discharge summaries, even in scenarios and contexts where a current template exists. Much of it depends on end-user data entry, review, and validation. The other options are minor challenges compared with accuracy of content.

\section{Protection of Human and Animal Subjects}

This study was performed in compliance with the World Medical Association Declaration of Helsinki on Ethical Principles for Medical Research Involving Human Subjects and was reviewed by RCH Human Research Ethics Committee (HREC \#DA001-2017-102).

\section{Conflict of Interest}

None.

\section{References}

1 Belleli E, Naccarella L, Pirotta M. Communication at the interface between hospitals and primary care - a general practice audit of hospital discharge summaries. Aust Fam Physician 2013;42(12): 886-890

2 Embi PJ, Yackel TR, Logan JR, Bowen JL, Cooney TG, Gorman PN. Impacts of computerized physician documentation in a teaching hospital: perceptions of faculty and resident physicians. J Am Med Inform Assoc 2004;11(04):300-309

3 Alderton M, Callen J. Are general practitioners satisfied with electronic discharge summaries? Health Inf Manag 2007;36(01):7-12

4 Forster AJ, Clark HD, Menard A, et al. Adverse events among medical patients after discharge from hospital. CMAJ 2004;170 (03):345-349

5 Chan S, Maurice AP, Pollard CW, Ayre SJ, Walters DL, Ward HE. Improving the efficiency of discharge summary completion by linking to preexisiting patient information databases. BMJ Qual Improv Rep 2014;3(01):u200548.w2006

6 Hahn JS, Bernstein JA, McKenzie RB, King BJ, Longhurst CA. Rapid implementation of inpatient electronic physician documentation at an academic hospital. Appl Clin Inform 2012;3(02): 175-185

7 Australian Commission on Safety and Quality in Healthcare. National guidelines for on-screen presentation of discharge summaries. Sydney, NSW: Commonwealth Government of Australia; April 01 2016. Available at: https://www.safetyandquality.gov.au/ wp-content/uploads/2017/02/Guidelines-on-screen-presentation -of-discharge-summaries.pdf. Accessed August 14, 2018

8 O'Leary KJ, Liebovitz DM, Feinglass J, Liss DT, Baker DW. Outpatient physicians' satisfaction with discharge summaries and perceived need for an electronic discharge summary. J Hosp Med 2006;1(05):317-320

9 Kripalani S, LeFevre F, Phillips CO, Williams MV, Basaviah P, Baker DW. Deficits in communication and information transfer between hospital-based and primary care physicians: implications for patient safety and continuity of care. JAMA 2007;297(08): 831-841

10 Wilson S, Ruscoe W, Chapman M, Miller R. General practitionerhospital communications: a review of discharge summaries. J Qual Clin Pract 2001;21(04):104-108

11 Schabetsberger T, Ammenwerth E, Andreatta S, et al. From a paper-based transmission of discharge summaries to electronic communication in health care regions. Int J Med Inform 2006;75 (3-4):209-215
12 O'Leary KJ, Liebovitz DM, Feinglass J, et al. Creating a better discharge summary: improvement in quality and timeliness using an electronic discharge summary. J Hosp Med 2009;4(04):219-225

13 Australian Commission on Safety and Quality in Healthcare. Electronic Discharge Summary Systems Literature Scan. Sydney, NSW: Commonwealth Government of Australia; August 2 2010. Available at: https://www.safetyandquality.gov.au/wp-content/uploads/2012 /02/e-DischargeLiteratureScan.pdf. Accessed August 14, 2017

14 Patel VL, Kushniruk AW, Yang S, Yale JF. Impact of a computer-based patient record system on data collection, knowledge organization, and reasoning. J Am Med Inform Assoc 2000;7(06):569-585

15 van Walraven C, Rokosh $\mathrm{E}$. What is necessary for high-quality discharge summaries? Am J Med Qual 1999;14(04):160-169

16 Buckler LT, Teasdale C, Turner M, Schadler A, Schwieterman TM, Campbell CL. The patient-centered discharge-an electronic discharge process is associated with improvements in quality and patient satisfaction. J Healthc Qual 2017;39(03):136-143

17 Rosenbloom ST, Crow AN, Blackford JU, Johnson KB. Cognitive factors influencing perceptions of clinical documentation tools. J Biomed Inform 2007;40(02):106-113

18 Coghlin DT, Leyenaar JK, Shen M, et al. Pediatric discharge content: a multisite assessment of physician preferences and experiences. Hosp Pediatr 2014;4(01):9-15

19 Leyenaar JK, Bergert L, Mallory LA, et al. Pediatric primary care providers' perspectives regarding hospital discharge communication: a mixed methods analysis. Acad Pediatr 2015;15(01):61-68

20 Allen-Graham J, Mitchell L, Heriot N, et al. Electronic health records and online medical records: an asset or a liability under current conditions? Aust Health Rev 2018;42(01):59-65

21 Snow V, Beck D, Budnitz T, et al; American College of Physicians; Society of General Internal Medicine; Society of Hospital Medicine; American Geriatrics Society; American College of Emergency Physicians; Society of Academic Emergency Medicine. Transitions of care consensus policy statement: American College of Physicians-Society of General Internal Medicine-Society of Hospital Medicine-American Geriatrics Society-American College of Emergency Physicians-Society of Academic Emergency Medicine. J Gen Intern Med 2009;24(08):971-976

22 Hammad EA, Wright DJ, Walton C, Nunney I, Bhattacharya D. Adherence to UK national guidance for discharge information: an audit in primary care. Br J Clin Pharmacol 2014;78(06):1453-1464

23 Unnewehr M, Schaaf B, Marev R, Fitch J, Friederichs H. Optimizing the quality of hospital discharge summaries-a systematic review and practical tools. Postgrad Med 2015;127(06):630-639

24 Australian Digital Health Agency. My Health Record; 2017. Available at: https://www.digitalhealth.gov.au/get-started-with-digital-health /what-is-digital-health/features-of-the-my-health-record-system/ my-health-record-system-healthcare-identifiers. Accessed August 20, 2017

25 Fathi R, Davis A, Campbell K. Discharge summaries for hospital to intermediate care facility transitions: assessing timeliness and quality of documentation by Internal Medicine residents. J Am Geriatr Soc 2013;(61):S107

26 Callen J, McIntosh J, Li J. Accuracy of medication documentation in hospital discharge summaries: a retrospective analysis of medication transcription errors in manual and electronic discharge summaries. Int J Med Inform 2010;79(01):58-64

27 Walz SE, Smith M, Cox E, Sattin J, Kind AJ. Pending laboratory tests and the hospital discharge summary in patients discharged to sub-acute care. J Gen Intern Med 2011;26(04):393-398

28 Hart C, Stevens T, Girling N. Are our discharge summaries fit for purpose? Palliat Med 2016;30(06):NP212-NP213

29 Boxer R, Gandara E, Schnipper J. Introduction of a discharge template to improve discharge documentation for patients with heart failure. J Hosp Med 2010;5(S1). Available at: https://www. shmabstracts.com/abstract/introduction-of-a-discharge-templateto-improve-discharge-documentation-for-patients-with-heart-failure/. Accessed August 31, 2018 
30 Key-Solle M, Paulk E, Bradford K, Skinner AC, Lewis MC, Shomaker $\mathrm{K}$. Improving the quality of discharge communication with an educational intervention. Pediatrics 2010;126(04):734-739

31 Grimes T, Fitzsimons M, Galvin M, Delaney T, Flanagan S. Evaluation of dispensing records from the Primary Care Reimbursement Services as a source of preadmission medication information. Pharmacoepidemiol Drug Saf 2011;20(06):663

32 Davis T. Paediatric prescribing errors. Arch Dis Child 2011;96(05): 489-491

33 Gattari TB, Krieger LN, Hu HM, Mychaliska KP. Medication discrepancies at pediatric hospital discharge. Hosp Pediatr 2015;5(08):439-445

34 Moore C, Wisnivesky J, Williams S, McGinn T. Medical errors related to discontinuity of care from an inpatient to an outpatient setting. J Gen Intern Med 2003;18(08):646-651

35 Li JY, Yong TY, Hakendorf P, Ben-Tovim D, Thompson CH. Timeliness in discharge summary dissemination is associated with patients' clinical outcomes. J Eval Clin Pract 2013;19(01):76-79
36 Feigenbaum P, Neuwirth E, Trowbridge L, et al. Factors contributing to all-cause 30-day readmissions: a structured case series across 18 hospitals. Med Care 2012;50(07):599-605

37 Tsou AY, Lehmann CU, Michel J, Solomon R, Possanza L, Gandhi T. Safe practices for copy and paste in the EHR. Systematic review, recommendations, and novel model for health IT collaboration. Appl Clin Inform 2017;8(01):12-34

38 Adhiyaman V, Oke A, White AD, Shah IU. Diagnoses in discharge communications: how far are they reliable? Int J Clin Pract 2000; 54(07):457-458

39 Bowman S. Impact of electronic health record systems on information integrity: quality and safety implications. Perspect Health Inf Manag 2013;10:1c

40 Dean SM, Gilmore-Bykovskyi A, Buchanan J, Ehlenfeldt B, Kind AJ. Design and hospitalwide implementation of a standardized discharge summary in an electronic health record. Jt Comm J Qual Patient Saf 2016;42(12):555-AP11

Appendix A Literature review search terms

\begin{tabular}{|l|}
\hline Medline Ovid \\
\hline "Discharge Summary" and "Gold Standard" $=9$ \\
\hline "Discharge Summary" and "Variability OR \\
Inconsistency" $=6$
\end{tabular}

Note: Keyword terms: Discharge Summary, Gold Standard, Template, Variability, Inconsistency. 